\title{
A CHARACTERIZATION OF MORITA EQUIVALENCE PAIRS
}

\author{
I. G. TODOROV \\ Department of Mathematics, University of the Aegean, 83200 Karlovassi, Samos, Greece ${ }^{1}$ \\ e-mail:ivan@aegean.gr
}

(Received 17 August, 2001; accepted 19 June, 2001)

\begin{abstract}
We characterize the pairs of operator spaces that occur as pairs of Morita equivalence bimodules between non-selfadjoint operator algebras in terms of the mutual relation between the spaces. We obtain a characterization of the operator spaces which are completely isometrically isomorphic to imprimitivity bimodules between some strongly Morita equivalent (in the sense of Rieffel) $\mathrm{C}^{*}$-algebras. As corollaries, we give representation results for such operator spaces.
\end{abstract}

2000 Mathematics Subject Classification. Primary 47L25; Secondary 47L30, 46L07.

1. Introduction and preliminaries. The notion of Morita equivalence is fundamental in both Algebra and Analysis. This notion entered the Theory of Operator Algebras with Rieffel's paper [5]. In that work, Rieffel defined Morita equivalence for $\mathrm{C}^{*}$ - and $\mathrm{W}^{*}$-algebras and proved various results concerning these notions. After the formulation of the theory of (abstract) operator spaces (see [4] for a survey and references), this notion was generalized [2] for non-selfadjoint operator algebras and obtained an important place in the theory. In this paper we give a characterization of those pairs $(X, Y)$ of operator spaces, which occur as pairs of Morita equivalence bimodules between non-selfadjoint (approximately unital) operator algebras, in terms of the mutual relation between the spaces $X$ and $Y$. We specialize our result to obtain a characterization of the operator spaces which are completely isometrically isomorphic to imprimitivity bimodules between strongly Morita equivalent (in the sense of Rieffel [5]) C*-algebras. We call these (abstract) operator spaces ternary operator systems. The Banach space analogue of these spaces, known as ternary $C^{*}$-rings have been studied by Zettl [6] and others. Our result is an operator space version of an implicit characterization of the imprimitivity bimodules between $\mathrm{C}^{*}$-algebras up to a Banach space isometry, contained in [6]. According to our result, additional conditions must be imposed on an operator space which is also a ternary $\mathrm{C}^{*}$-ring, in order to be completely isometric to a Morita equivalence bimodule between some $\mathrm{C}^{*}$-algebras. Thus, the similarity beween ternary $\mathrm{C}^{*}$-rings and ternary operator systems is the associativity property of the triple product; the difference is that the " $\mathrm{C}^{*}$-condition" for a ternary operator system takes into account its operator space structure as well (see Definition 4).

Finally, we point out versions of the Representation Theorem of [6] for the case of a ternary operator system.

In order to state and prove our results, we recall some notions and notation. We have as a main reference the monograph [4]. An operator space is a Banach space $X$

${ }^{1}$ Current address: Department of Pure Mathematics, Queens University Belfast, Belfast BT7 1NN, U.K. e-mail: i.todorov@qub.ac.uk 
endowed with a sequence of norms on the linear space $M_{n}(X)$ of $n \times n$ matrices with entries in $X$ for which the (left and right) actions of $M_{n}(\mathbb{C})$ on $M_{n}(X)$ are contractive and the norm of a direct sum of two matrices equals the maximum of their norms. A map $\varphi: X \longrightarrow Y$ between the operator spaces $X$ and $Y$ is said to be completely bounded (contractive, isometric), if the map $\varphi_{n}: M_{n}(X) \longrightarrow M_{n}(Y)$ given by $\varphi_{n}\left(\left(x_{i j}\right)\right)=\left(\varphi\left(x_{i j}\right)\right)$ (where $\left.\left(x_{i j}\right) \in M_{n}(X)\right)$ is bounded (contractive, isometric) for each $n \in \mathbb{N}$ and $\|\varphi\|_{c b}=\sup _{n \in \mathbb{N}}\left\|\varphi_{n}\right\|$ is finite. If $x=\left(x_{i j}\right), y=\left(y_{i j}\right) \in M_{n}(X)$, then we let $x \odot y=\left(\sum_{k} x_{i k} \otimes y_{k j}\right)_{i, j} \in M_{n}(X \otimes Y)$. If an operator space $X$ is an $A, B$-bimodule, where $A$ and $B$ are algebras, we define $a \odot x=\left(\sum_{k} a_{i k} \cdot x_{k j}\right)_{i, j}$ and $x \odot b=$ $\left(\sum_{k} x_{i k} \cdot b_{k j}\right)_{i, j}$, where $a=\left(a_{i j}\right) \in M_{n}(A)$ and $b=\left(b_{i j}\right) \in M_{n}(B)$. The Haagerup tensor product of the operator spaces $X$ and $Y$ can be described by defining a norm $\|\cdot\|_{n}$ on $M_{n}(X \otimes Y)$ by setting

$$
\|U\|_{n}=\inf \left\{\|x\|\|y\|: U=x \odot y, x \in M_{n, p}(X), y \in M_{p, n}(Y), p \in \mathbb{N}\right\}
$$

for each $U \in M_{n}(X \otimes Y)$ and $n \in \mathbb{N}$ and letting $X \otimes_{h} Y$ be the completion of $X \otimes Y$ with respect to $\|\cdot\|_{1}$. The Haagerup tensor product is associative. Moreover, if $X_{1}, X_{2}, Y_{1}$ and $Y_{2}$ are operator spaces and $f_{1}: X_{1} \rightarrow Y_{1}$ and $f_{2}: X_{2} \longrightarrow Y_{2}$ are completely bounded maps, then there is a unique completely bounded map $f_{1} \otimes f_{2}$ : $X_{1} \otimes_{h} X_{2} \longrightarrow Y_{1} \otimes_{h} Y_{2}$ such that $f_{1} \otimes f_{2}\left(x_{1} \otimes x_{2}\right)=f_{1}\left(x_{1}\right) \otimes f_{2}\left(x_{2}\right), x_{1} \in X_{1}, x_{2} \in X_{2}$. We have that $\left\|f_{1} \otimes f_{2}\right\|_{c b} \leq\left\|f_{1}\right\|_{c b}\left\|f_{2}\right\|_{c b}$. Each multilinear completely bounded map from the direct product of several operator spaces into an operator space can be linearized through the Haagerup tensor product of its domain spaces (see [3] for the theory of multilinear completely bounded maps). An approximately unital Banach algebra will be a Banach algebra which is not unital but which possesses a contractive approximate identity. We assume that the norm of the unit in a unital Banach algebra is 1. If an approximately unital or unital Banach algebra is an operator space and its multiplication is completely contractive (we call such an object an (abstract) operator algebra), then it is completely isometrically isomorphic to an algebra of operators on a Hilbert space. Two approximately unital or unital operator algebras $A$ and $B$ are said to be Morita equivalent if there exist operator spaces $X$ and $Y$, such that

(1) $X$ is an $A, B$-bimodule, $Y$ is a $B, A$-bimodule with completely contractive module actions;

(2) there are completely bounded bimodule maps $(\cdot, \cdot): X \times Y \rightarrow A$ and $[\cdot, \cdot]: Y \times X \longrightarrow B$ such that $(x \cdot b, y)=(x, b \cdot y),[y \cdot a, x]=[y, a \cdot x]$ (that is, these maps are balanced $),\left(x_{1}, y\right) \cdot x_{2}=x_{1} \cdot\left[y, x_{2}\right],\left[y_{1}, x\right] \cdot y_{2}=y_{1} \cdot\left(x, y_{2}\right)$ for each $x, x_{1}, x_{2} \in X, y, y_{1}, y_{2} \in Y, a \in A, b \in B$ and

(3) the linearized maps on the Haagerup tensor product induced by the pairings are complete quotients (see [2] for the exact definition of this condition). The 6-tuple $(A, B, X, Y,(\cdot, \cdot),[\cdot, \cdot])$ is called a Morita context.

If $X$ is a Banach space, we denote by $B(X)$ the (Banach) algebra of bounded linear operators on $X$.

2. Morita pairs between non-selfadjoint operator algebras. In this section we characterize Morita pairs between non-selfadjoint approximately unital or unital operator algebras. We give a full proof of the result only in the case in which both algebras are approximately unital. We then comment on the necessary changes to be made if one (both) of the algebras is (are) unital. 
Theorem 1. Let $X$ and $Y$ be operator spaces. Then $(X, Y)$ is a Morita equivalence pair between some approximately unital operator algebras if and only if there exist completely bounded maps $p: X \otimes_{h} Y \otimes_{h} X \rightarrow X$ and $q: Y \otimes_{h} X \otimes_{h} Y \rightarrow Y$ and nets $\left\{e_{\alpha}\right\} \subset X \otimes Y$ and $\left\{f_{\beta}\right\} \subset Y \otimes X$ with $\left\|e_{\alpha}\right\|_{c b}<1$ and $\left\|f_{\beta}\right\|_{c b}<1$ such that

(1) $p\left(p\left(x_{1} \otimes y_{1} \otimes x_{2}\right) \otimes y_{2} \otimes x_{3}\right)=p\left(x_{1} \otimes q\left(y_{1} \otimes x_{2} \otimes y_{2}\right) \otimes x_{3}\right)$

$=p\left(x_{1} \otimes y_{1} \otimes p\left(x_{2} \otimes y_{2} \otimes x_{3}\right)\right)$;

(2) $q\left(q\left(y_{1} \otimes x_{1} \otimes y_{2}\right) \otimes x_{2} \otimes y_{3}\right)=q\left(y_{1} \otimes p\left(x_{1} \otimes y_{2} \otimes x_{2}\right) \otimes y_{3}\right)$

$=q\left(y_{1} \otimes x_{1} \otimes q\left(y_{2} \otimes x_{2} \otimes y_{3}\right)\right)$;

(3) $\lim _{\alpha} p\left(e_{\alpha} \otimes x\right)=x$ and

(4) $\lim _{\beta} q\left(f_{\beta} \otimes y\right)=y$

for each $x, x_{i} \in X$ and $y, y_{i} \in Y(i=1,2,3)$. Moreover, the pairings in the respective Morita context are completely contractive if and only if the mappings $p$ and $q$ with the properties (1)-(4) can be chosen to be completely contractive.

We shall give two proofs of the above theorem. The first one is applicable only for completely contractive pairings, while the second one can be applied in the general case.

First proof. Let $X$ and $Y$ be operator spaces, $p: X \otimes_{h} Y \otimes_{h} X \rightarrow X$ and $q: Y \otimes_{h} X \otimes_{h} Y \rightarrow Y$ completely contractive maps that satisfy conditions (1)-(4) for some nets $\left\{e_{\alpha}\right\} \subset X \otimes Y$ and $\left\{f_{\beta}\right\} \subset Y \otimes X$ with $\left\|e_{\alpha}\right\|_{c b}<1$ and $\left\|f_{\beta}\right\|_{c b}<1$. We divide the proof into several steps.

Step 1. Let $A_{1}=X \otimes_{h} Y$. For each $a \in A_{1}$ let $L_{a}: X \rightarrow X$ be the map given by $L_{a}(x)=p(a \otimes x)$. It is clear that $L_{a}$ is completely contractive. Let $A_{0}=\left\{a \in A_{1}\right.$ : $\left.L_{a}=0\right\}$; it is obvious that $A_{0}$ is a closed subspace of $A_{1}$. We define a multiplication $m$ on $A_{1}$ as follows. If $a \in A_{1}$ is an arbitrary element and $b=\sum_{i} x_{i} \otimes y_{i} \in A_{1}$ is a finite sum of elementary tensors, then we let $m(a, b)=\sum_{i} p\left(a \otimes x_{i}\right) \otimes y_{i}$. We first prove associativity: if $a, b=\sum_{i} x_{i} \otimes y_{i}, c=\sum_{j} u_{j} \otimes v_{j} \in A_{1}$, then

$$
\begin{aligned}
m(a, m(b, c)) & =m\left(a, \sum_{i, j} p\left(x_{i} \otimes y_{i} \otimes u_{j}\right) \otimes v_{j}\right) \\
& =\sum_{i, j} p\left(a \otimes p\left(x_{i} \otimes y_{i} \otimes u_{j}\right)\right) \otimes v_{j} \\
& =\sum_{i, j} p\left(p\left(a \otimes x_{i}\right) \otimes y_{i} \otimes u_{j}\right) \otimes v_{j} \\
& =\sum_{j} p\left(m(a, b) \otimes u_{j}\right) \otimes v_{j}=m(m(a, b), c) .
\end{aligned}
$$

By the associativity of the Haagerup tensor product we have that the map $m$ coincides with the map

$$
p \otimes \mathrm{id}:\left(A_{1} \otimes_{h} X\right) \otimes_{h} Y \longrightarrow X \otimes_{h} Y .
$$

The last map is completely contractive as a tensor product of completely contractive maps and so $m$ is completely contractive. It follows that $m$ can be extended to all of $A_{1} \times A_{1}$ and the extention, which will be denoted again by $m$, is associative. Condition (3) implies that $\left\{e_{\alpha}\right\}$ is an approximate unit for $A_{1}$. It follows from [1] that $A_{1}$ is 
an (abstract) operator algebra. Next we point out that $A_{0}$ is a (two-sided) ideal in $A_{1}$. First observe that for each $a, b \in A_{1}$ and $z \in X$ we have

$$
p(m(a, b) \otimes z)=p(a \otimes p(b \otimes z)) .
$$

Indeed, if $a=x_{1} \otimes y_{1}$ and $b=x_{2} \otimes y_{2}$ are elementary tensors, then by (1) we have

$$
\begin{aligned}
p(m(a, b) \otimes z) & =p\left(p\left(x_{1} \otimes y_{1} \otimes x_{2}\right) \otimes y_{2} \otimes z\right) \\
& =p\left(x_{1} \otimes y_{1} \otimes p\left(x_{2} \otimes y_{2} \otimes z\right)\right)=p(a \otimes p(b \otimes z)),
\end{aligned}
$$

and the general case follows by (bi)linearity and approximation. Now if $a \in A_{0}$, $x \otimes y \in A_{1}$ and $z \in X$, then

$$
L_{m(a, x \otimes y)}(z)=L_{p(a \otimes x) \otimes y}(z)=p(p(a \otimes x) \otimes y \otimes z)=0
$$

and, using (1),

$$
L_{m(x \otimes y, a)}(z)=p(m(x \otimes y, a) \otimes z)=p(x \otimes y \otimes p(a \otimes z))=0 .
$$

This shows that $A_{0}$ is an ideal in $A_{1}$. Let $A=A_{1} / A_{0}$; by [1] we have that $A$ is an (abstract) operator algebra. Similarly we define a multiplication on $B_{1}=Y \otimes_{h} X$ that turns $B_{1}$ into an (abstract) operator algebra, completely bounded maps $R_{b}: Y \rightarrow Y, b \in B_{1}$, and an ideal $B_{0} \subset B_{1}$. Let $B=B_{1} / B_{0}$ be the quotient operator algebra.

Step 2. We equip the spaces $X$ and $Y$ with structures of $A, B$ - and $B, A$-bimodules respectively. If $a \in A_{1}$ and $x \in X$, let

$$
\left(a+A_{0}\right) \cdot x=p(a \otimes x) .
$$

If $b \in A_{1}$ is such that $a-b \in A_{0}$, then by the definition of $A_{0}$ we have that $p((a-b) \otimes x)=0$, which implies that the left action of $A$ on $X$ is well defined. From the fact that $p$ is completely contractive it follows that this action is completely contractive as well. The right action of $B$ on $X$ is defined by setting, for each $b \in B$ and $x \in X$,

$$
x \cdot\left(b+B_{0}\right)=p(x \otimes b) .
$$

To prove that this is a well defined action, suppose that $b \in B_{0}$; that is, $q(b \otimes y)=0$ for each $y \in Y$. If $x_{1} \in X, y_{1} \in Y$, we have that

$$
p\left(p(x \otimes b) \otimes y_{1} \otimes x_{1}\right)=p\left(x \otimes q\left(b \otimes y_{1}\right) \otimes x_{1}\right)=0
$$

and thus $p(p(x \otimes b) \otimes d)=0$ for each $d \in Y \otimes_{h} X$ which, according to (4), implies that $p(x \otimes b)=0$. It follows that the right action of $B$ on $X$ is well defined and, as before, completely contractive. In other words, $X$ is endowed with a structure of an $A, B$-operator bimodule. The $A, B$-operator bimodule structure of the operator space $Y$ is defined by letting $>$

$$
\left(b+B_{0}\right) \cdot y=q(b \otimes y)
$$


and

$$
y \cdot\left(a+A_{0}\right)=q(y \otimes a)
$$

for each $a \in A_{1}, b \in B_{1}$ and $y \in Y$. By symmetry, these actions are well defined and completely contractive.

Step 3. We define pairings $(\cdot, \cdot): X \times Y \rightarrow A$ and $[\cdot, \cdot]: Y \times X \rightarrow B$ by letting $(x, y)=x \otimes y+A_{0}$ and $[y, x]=y \otimes x+B_{0}$. It is obvious that the pairings $(\cdot, \cdot)$ and $[\cdot, \cdot]$ are completely contractive.

Step 4. We show that the pairings defined in Step 3 are balanced bimodule maps. Indeed, if $b \in B_{1}, \quad x \in X$ and $y \in Y$, then by (1) we have that $p(p(x \otimes b) \otimes y \otimes z)=p(x \otimes q(b \otimes y) \otimes z)$, for each $z \in X$, which means that $p(x \otimes b) \otimes y-x \otimes q(b \otimes y) \in A_{0}$ and thus $\left(x \cdot\left(b+B_{0}\right), y\right)=\left(x,\left(b+B_{0}\right) \cdot y\right)$, for each $x \in X$ and $y \in Y$. Similarly one checks that the pairing [., $\cdot]$ is $A$-balanced.

Let $a_{1}=x_{1} \otimes y_{1}, a_{2}=x_{2} \otimes y_{2} \in A_{1}$ and $z \in X$. Then by (1) we have

$$
p\left(p\left(a_{1} \otimes x\right) \otimes q\left(y, a_{2}\right) \otimes z\right)=p\left(p\left(p\left(a_{1} \otimes x\right) \otimes y \otimes x_{2}\right) \otimes y_{2} \otimes z\right)
$$

which means that

$$
p\left(a_{1} \otimes x\right) \otimes q\left(y \otimes a_{2}\right)-p\left(p\left(a_{1} \otimes x\right) \otimes y \otimes x_{2}\right) \otimes y_{2} \in A_{0},
$$

for each $x \in X$ and $y \in Y$ and so $\left(a_{1} \cdot x, y \cdot a_{2}\right)=a_{1}(x, y) a_{2}$. Similarly, the pairing $[\cdot, \cdot]$ is a $B$-bimodule map.

Step 5. We have that $\left(x_{1}, y\right) \cdot x_{2}=x_{1} \cdot\left[y, x_{2}\right]$ and $\left[y_{1}, x\right] \cdot y_{2}=y_{1} \cdot\left(x, y_{2}\right)$ for each $x_{1}, x_{2} \in X$ and $y_{1}, y_{2} \in Y$. These identities are direct consequences of the definitions of the pairings and the module actions.

Step 6. The projection maps $\pi: A_{1} \rightarrow A$ and $\rho: B_{1} \rightarrow B$ are complete quotients. This is an immediate consequence of conditions (3) and (4) and Lemma 2.9. of [2].

Steps 1-6 above ensure that $(A, B, X, Y,(\cdot, \cdot),[\cdot, \cdot])$ is a Morita context with completely contractive pairings.

For the converse direction, suppose that $(A, B, X, Y,(\cdot, \cdot),[\cdot, \cdot])$ is a Morita context with completely contractive pairings. Define $\tilde{p}: X \times Y \times X \rightarrow X$ and $\tilde{q}: Y \times X \times Y \rightarrow Y$ by letting

$$
\tilde{p}\left(x_{1}, y, x_{2}\right)=\left(x_{1}, y\right) \cdot x_{2}
$$

and

$$
\tilde{q}\left(y_{1}, x, y_{2}\right)=\left(y_{1}, x\right) \cdot y_{2} \text {. }
$$

It is obvious that $\tilde{p}$ and $\tilde{q}$ are completely contractive trilinear maps. If $p: X \otimes_{h} Y \otimes_{h} X \rightarrow X$ and $q: Y \otimes_{h} X \otimes_{h} Y \rightarrow Y$ are their linearizations through the Haagerup tensor product, then it is easily seen from the definition of a Morita context that Properties (1), (2) (3) and (4) hold. 
Second proof. Assume that $X$ and $Y$ are operator spaces, $p: X \otimes_{h} Y \otimes_{h} X \rightarrow X$ and $q: Y \otimes_{h} X \otimes_{h} Y \rightarrow Y$ completely contractive maps which satisfy conditions (1)(4) for some families $\left\{e_{\alpha}\right\} \subset X \otimes Y$ and $\left\{f_{\beta}\right\} \subset Y \otimes X$ with $\left\|e_{\alpha}\right\|_{c b}<1$ and $\left\|f_{\beta}\right\|_{c b}<1$. We recall that $L_{a}: X \rightarrow X$ and $R_{b}: Y \rightarrow Y$ are the operators given by $L_{a}(x)=$ $p(a \otimes x)$ and $R_{b}(y)=q(b \otimes y)$, where $a \in X \otimes_{h} Y$ and $b \in Y \otimes_{h} X$. Further $L_{a} \in B(X)$ and $R_{b} \in B(Y)$. Let $\mathcal{L}=\left\{L_{a}: a \in X \otimes_{h} Y\right\}^{-}$and $\mathcal{R}=\left\{R_{b}: b \in Y \otimes_{h} X\right\}^{-}$, the closures being taken in the norm topology of $B(X)$. As in the first proof, we shall only point out the "left" considerations in constructing the Morita context whenever the "right" ones follow either by symmetry or in a similar way. First note that $\mathcal{L}$ is a subalgebra of $B(X)$. Indeed, we have that

$$
\begin{aligned}
L_{x_{1} \otimes y_{1}} L_{x_{1} \otimes y_{1}}(z) & =p\left(x_{1} \otimes y_{1} \otimes p\left(x_{2} \otimes y_{2} \otimes z\right)\right. \\
& =p\left(p\left(x_{1} \otimes y_{1} \otimes x_{2}\right) \otimes y_{2} \otimes z\right)=L_{p\left(x_{1} \otimes y_{1} \otimes x_{2}\right) \otimes y_{2}}(z),
\end{aligned}
$$

for each $z \in X$, and it follows that $L_{a} L_{b} \in \mathcal{L}$ whenever $a, b \in X \otimes_{h} Y$. Next observe that $\mathcal{L}$ is approximately unital according to (3). Indeed, if $z \in X,\|z\| \leq 1$ and $a=x \otimes y \in X \otimes_{h} Y$, then

$$
\begin{aligned}
\left\|L_{e_{\alpha}} L_{a}(z)-L_{a}(z)\right\| & =\left\|p\left(p\left(e_{\alpha} \otimes x\right) \otimes y \otimes z\right)-p(x \otimes y \otimes z)\right\| \\
& =\left\|p\left(\left(p\left(e_{\alpha} \otimes x\right)-x\right) \otimes y \otimes z\right)\right\| \leq\left\|p\left(e_{\alpha} \otimes x\right)-x\right\|\|y\|
\end{aligned}
$$

and clearly $\left\|p\left(e_{\alpha} \otimes x\right)-x\right\|\|y\| \longrightarrow_{\alpha} 0$.

Endow the operator space $X$ with the structure of an $\mathcal{L}, \mathcal{R}$-bimodule, by letting $L_{a} \cdot x=p(a \otimes x)$ and $x \cdot R_{b}=p(x \otimes b)$. In a similar way, endow the operator space $Y$ with the structure of an $\mathcal{R}, \mathcal{L}$-bimodule.

Let $n \in \mathbb{N}$. We define a norm $\|\cdot\|_{n}$ on $M_{n}(\mathcal{L})$, letting, for an element $L=\left(L_{a_{i j}}\right) \in M_{n}(\mathcal{L}),\|L\|_{n}$ to be the maximum of the numbers

$$
\sup \left\{\|L \odot x\|_{n}: x=\left(x_{i j}\right) \in M_{n}(X),\|x\|_{n} \leq 1\right\}
$$

and

$$
\sup \left\{\|y \odot L\|_{n}: y=\left(y_{i j}\right) \in M_{n}(Y),\|y\|_{n} \leq 1\right\}
$$

it is clear that $\|L\|_{n} \leq\|p\|_{n}$. It is easy to observe that the sequence $\{\|\cdot\|\}_{n \in \mathbb{N}}$ defines an operator space structure on $\mathcal{L}$. We show that $\mathcal{L}$ is actually an operator algebra with respect to this operator space structure by checking that the multiplication on $\mathcal{L}$ is completely contractive. Indeed, if $\left(T_{i j}\right),\left(S_{i j}\right) \in M_{n}(\mathcal{L})$, then

$$
\left\|\left(T_{i j}\right) \odot\left(S_{i j}\right) \odot\left(x_{i j}\right)\right\|_{n}=\left\|\left(\sum_{k, l} T_{i k}\left(S_{k l}\left(x_{l j}\right)\right)\right)_{i, j}\right\|_{n} \leq\left\|\left(T_{i j}\right)\right\|_{n}\left\|\left(S_{i j}\right)\right\|_{n}\left\|\left(x_{i j}\right)\right\|_{n}
$$

and, similarly, $\|\left(\left(y_{i j}\right) \odot\left(T_{i j}\right) \odot\left(S_{i j}\right)\|\leq\|\left(T_{i j}\right)\left\|_{n}\right\|\left(S_{i j}\right)\left\|_{n}\right\|\left(y_{i j}\right) \|_{n}\right.$, which proves the assertion. It follows that $\mathcal{L}$ is an operator algebra with respect to the defined sequence of matrix norms. Similarly, $\mathcal{R}$ is an operator algebra with respect to an analogous sequence of matrix norms. From the definition of the matrix norms of $\mathcal{L}$ it follows that the module actions of $\mathcal{R}$ and $\mathcal{L}$ on $X$ and $Y$ are completely contractive.

Define pairings $(\cdot, \cdot): X \times Y \rightarrow \mathcal{L}$ and $[\cdot, \cdot]: Y \times X \rightarrow \mathcal{R}$ by setting $(x, y)=L_{x \otimes y}$ and $[y, x]=R_{y \otimes x}$, where $x \in X$ and $y \in Y$. The fact that the pairings are balanced 
bimodule maps and the "linking" properties between the two pairings are verified readily as in the first proof.

The converse direction is established as in the first proof.

In the case in which one (both) of the algebras in the Morita context is (are) unital, some changes in conditions (3) or (and) (4) in Theorem 1 must be made. For example, suppose that we want to characterize those pairs $(X, Y)$ of operator spaces for which there exist operator algebras $A$ and $B$ such that $A$ is unital, $B$ is approximately unital and $A, B$ are Morita equivalent with an equivalence pair $(X, Y)$. Then, from Lemma 2.8 in [2] it follows that the existence of the family $\left\{e_{\alpha}\right\}$ and condition (3) must be replaced by the following condition.

(3') For each $\epsilon>0$ there exists an element $e_{\epsilon} \in X \otimes_{h} Y$ such that $\left\|e_{\epsilon}\right\|<1+\epsilon$ and $p\left(e_{\epsilon} \otimes x\right)=x$ for each $x \in X$.

Similar changes must be made in the characterizations of Morita pairs $(X, Y)$ between some algebras $A$ and $B$ when both $A$ and $B$ are unital or $A$ is approximately unital and $B$ is unital.

Theorem 1 has the following immediate consequence.

COROllary 2. Let $X$ and $Y$ be operator spaces with the properties (1)-(4) of Theorem 1. Then there exist completely isometric representations $\varphi: X \rightarrow \mathcal{B}(\mathcal{H}, \mathcal{K})$ and $\psi: Y \rightarrow \mathcal{B}(\mathcal{K}, \mathcal{H})$ (where $\mathcal{H}$ and $\mathcal{K}$ are Hilbert spaces) such that $\varphi(X) \psi(Y) \varphi(X) \subseteq$ $\varphi(X)$ and $\psi(Y) \varphi(X) \psi(Y) \subseteq \psi(Y)$.

3. The selfadjoint case. In this section we consider the consequences of Theorem 1 when the operator algebras in the Morita context are $\mathrm{C}^{*}$-algebras. If $\left(X,\left\{\|\cdot\|_{n}\right\}\right.$ is an operator space, then we are able to form its conjugate space $X^{*}$. As a group with respect to the addition, $X^{*}$ coincides with $X$, the scalar multiplication is given by $\lambda x^{*}=(\bar{\lambda} x)^{*}$ (where $x^{*}$ is $x$ viewed as an element of $X^{*}$ ). The operator space structure on $X^{*}$ is given by assigning to an $n \times n$ matrix $\left(x_{i j}^{*}\right)_{i, j}$ with entries in $X^{*}$ the norm $\left\|\left(x_{j i}\right)_{i, j}\right\|_{n}$. Note that, with respect to these norms, there is a natural identification between $M_{n}\left(X^{*}\right)$ and $M_{n}(X)^{*}$.

Recall that an imprimitivity bimodule between two $\mathrm{C}^{*}$-algebras $A$ and $B$ is a Banach space $X$ which is an $A, B$-bimodule and which is equipped with sesquilinear forms ${ }_{A}\langle\cdot, \cdot\rangle: X \times X \rightarrow A$ and $\langle\cdot, \cdot\rangle_{B}: X \times X \rightarrow B$, such that ${ }_{A}\langle\cdot, \cdot\rangle$ is conjugate linear on the second variable, $\langle\cdot, \cdot\rangle_{B}$ is conjugate linear on the first, $\left(A, X,,_{A}\langle\cdot, \cdot\rangle\right)$ and $\left(B, X,\langle\cdot, \cdot\rangle_{B}\right)$ are Hilbert $A$-modules, ${ }_{A}\langle x, y\rangle z=x\langle y, z\rangle_{B}$, and the linear spans of $\left\{{ }_{A}\langle x, y\rangle: x, y \in X\right\}$ and $\left\{\langle x, y\rangle_{B}: x, y \in X\right\}$ are dense in $A$ and $B$ respectively. If $A$ and $B$ are $C^{*}$-algebras and $X$ is an imprimitivity bimodule between $A$ and $B$, then we are able to form an imprimitivity $B, A$-bimodule $X^{*}$ in the natural way, letting ${ }_{B}\left\langle x^{*}, y^{*}\right\rangle=\langle y, x\rangle_{B}^{*}$ and $\left\langle x^{*}, y^{*}\right\rangle_{A}={ }_{A}\langle y, x\rangle^{*}$. The set

$$
\mathcal{C}=\left(\begin{array}{cc}
A & X \\
X^{*} & B
\end{array}\right)
$$

can be endowed with a norm turning it into a $\mathrm{C}^{*}$-algebra under the multiplication performed using the respective module actions and pairings. Since a $\mathrm{C}^{*}$-algbera has a canonical operator space structure, the inclusion 


$$
x \hookrightarrow\left(\begin{array}{ll}
0 & x \\
0 & 0
\end{array}\right)
$$

endows $X$ with a canonical operator space structure. We consider an imprimitivity bimodule as an operator space with respect to this canonical operator space structure.

Now we are ready to establish the following theorem.

Theorem 3. Let $X$ be an operator space. Then $X$ is (completely isometrically isomorphic to) an imprimitivity bimodule between certain strongly Morita equivalent (in the sense of Rieffel) $C^{*}$-algebras if and only if there exists a completely contractive map $p: X \otimes_{h} X^{*} \otimes_{h} X \rightarrow X$ such that

(a) $p\left(p\left(x_{1} \otimes x_{2}^{*} \otimes x_{3}\right) \otimes x_{4}^{*} \otimes x_{5}\right)=p\left(x_{1} \otimes p\left(x_{4} \otimes x_{3}^{*} \otimes x_{2}\right)^{*} \otimes x_{5}\right)=$ $p\left(x_{1} \otimes x_{2}^{*} \otimes p\left(x_{3} \otimes x_{4}^{*} \otimes x_{5}\right)\right)$ and

(b) $\left\|p_{n}\left(\left(x_{i j}\right) \odot\left(x_{i j}\right)^{*} \odot\left(x_{i j}\right)\right)\right\|_{n}=\left\|\left(x_{i j}\right)\right\|_{n}^{3}$, for each $n \in \mathbb{N}$.

Proof. Suppose that an operator space $X$ and a map $p$ are given such that (a) and (b) are satisfied. Let $q: X^{*} \otimes_{h} X \otimes_{h} X^{*} \rightarrow X^{*}$ be the map given by

$$
q\left(x^{*} \otimes y \otimes z^{*}\right)=p\left(z \otimes y^{*} \otimes x\right)^{*}, \quad x, y, z \in X .
$$

An immediate verification shows that $p$ and $q$ satisfy conditions (1) and (2) of Theorem 1. As in the second proof of Theorem 1 we construct the operator spaces $\mathcal{L}$ and $\mathcal{R}$, which are also algebras with a completely contractive multiplication. Note that we are not able to conclude directly that $\mathcal{L}$ and $\mathcal{R}$ are operator algebras since they are not a priori approximately unital or unital. We shall show, however, that $\mathcal{L}$ and $\mathcal{R}$ are $C^{*}$-algebras and that their operator space structure, whose sequence of matrix norms will be denoted by $\left\{\|\cdot\|_{n}\right\}$, coincides with their canonical $\mathrm{C}^{*}$-algebra operator space structure. Actually, we will directly show that $M_{n}(\mathcal{L})$ (and so $M_{n}(\mathcal{R})$ as well) is a $\mathrm{C}^{*}$-algebra with respect to $\|\cdot\|_{n}$. First we define involutions on $\mathcal{L}$ and $\mathcal{R}$ : if $x, y \in X$, we let $L_{x \otimes y^{*}}^{*}=L_{y \otimes x^{*}}$ and $R_{y^{*} \otimes x}^{*}=R_{x^{*} \otimes y}$. The fact that the mappings $L \rightarrow L^{*}$ and $R \rightarrow R^{*}$ are indeed involutions on $\mathcal{L}$ and $\mathcal{R}$ follows easily from condition (a).

Let $a_{i j}=\sum_{u=1}^{v_{i j}} x_{i j}^{u} \otimes y_{i j}^{u *}, L=\left(L_{a_{i j}}\right)_{i, j} \in M_{n}(\mathcal{L})$ and $z=\left(z_{i j}\right)_{i, j} \in M_{n}(X)$. Then by (b) we have

$$
\begin{aligned}
\|L \odot z\|_{n}^{3} & =\left\|p_{n}\left(\left(\left(L_{a_{i j}}\right) \odot\left(z_{i j}\right)\right) \odot\left(\left(L_{a_{i j}}\right) \odot\left(z_{i j}\right)\right)^{*} \odot\left(\left(L_{a_{i j}}\right) \odot\left(z_{i j}\right)\right)\right)\right\| \\
& =\left\|p_{n}\left(\left(\sum_{k} p\left(a_{i k} \otimes z_{k j}\right)\right)_{i, j} \odot\left(\sum_{l} p\left(a_{t l} \otimes z_{l s}\right)^{*}\right)_{s, t} \odot\left(\sum_{m} p\left(a_{r m} \otimes z_{m v}\right)\right)_{r, v}\right)\right\| \\
& =\|\left(\left(\sum_{j, l, k, r, s, u} p\left(p\left(a_{i k} \otimes z_{k j}\right) \otimes p\left(a_{s l} \otimes z_{l j}\right)^{*} \otimes p\left(a_{s r} \otimes z_{r v}\right)\right)\right)_{i, v} \|_{n}\right. \\
& =\|\left(\sum_{j, l, k, r, s, u} p\left(p\left(a_{i k} \otimes z_{k j}\right) \otimes p\left(y_{s r}^{u} \otimes x_{s r}^{u *} \otimes p\left(a_{s l} \otimes z_{l j}\right)\right)^{*} \otimes z_{r v}\right)_{i, v} \|_{n} .\right.
\end{aligned}
$$

On the other hand, 


$$
\begin{aligned}
L^{*} \odot(L \odot z) & =\left(L_{a_{j i}}^{*}\right)_{i, j} \odot\left(\sum_{l} p\left(a_{s l} \otimes z_{l t}\right)\right)_{s, t} \\
& =\left(\sum_{s} L_{a_{s r}}^{*}\left(\sum_{l} p\left(a_{s l} \otimes z_{l t}\right)\right)\right)_{r, t} \\
& =\left(\sum_{s, l, u} p\left(y_{s r}^{u} \otimes x_{s r}^{u *} \otimes p\left(a_{s l} \otimes z_{l t}\right)\right)\right)_{r, t}
\end{aligned}
$$

and thus

$$
\begin{aligned}
p_{n}\left((L \odot z) \odot\left(L^{*} \odot(L \odot(z))\right)^{*} \odot z\right) & \\
& =p_{n}\left(\sum_{j, l, k, r, s, u} p\left(a_{i k} \otimes z_{k j}\right) \otimes p\left(y_{s r}^{u} \otimes x_{s r}^{u *} \otimes p\left(a_{s l} \otimes z_{l j}\right)\right)^{*} \otimes z_{r v}\right)_{i, v} .
\end{aligned}
$$

We conclude that

$$
\begin{aligned}
\|L \odot z\|_{n}^{3} & =\left\|p_{n}\left((L \odot z) \odot\left(L^{*} \odot(L \odot(z))\right)^{*} \odot z\right)\right\|_{n} \\
& \leq\|L \odot z\|_{n} \|\left(L^{*} \odot(L \odot(z))^{*}\left\|_{n}\right\| z \|_{n} .\right.
\end{aligned}
$$

Since the involution is isometric, it follows that

$$
\|L \odot z\|^{2} \leq\left\|\left(L^{*} \odot L\right) \odot z\right\| .
$$

Similarly, we obtain that

$$
\left\|z^{\prime} \odot L\right\|^{2} \leq\left\|z^{\prime} \odot\left(L^{*} \odot L\right)\right\|
$$

for each $z^{\prime} \in M_{n}(Y)$. It follows that

$$
\|L\|_{n}^{2} \leq\left\|L^{*} \odot L\right\|_{n}
$$

which implies that $\|\cdot\|_{n}$ is a $\mathrm{C}^{*}$-norm on $M_{n}(\mathcal{L})$. For $n=1$, we obtain that $\mathcal{L}$ is a $\mathrm{C}^{*}$-algebra. Since $M_{n}(\mathcal{L})$ has a unique $\mathrm{C}^{*}$-norm, it follows that $\|\cdot\|_{n}$ coincides with the norm on $M_{n}(\mathcal{L})$ "inherited" from the $\mathrm{C}^{*}$-structure of $\mathcal{L}$.

Thus we have shown that $(\mathcal{L}, \mathcal{R}, X, Y,(\cdot, \cdot),[\cdot, \cdot])$ is a Morita context. From Theorem 6.2 of [2] it follows that there exists a complete isometry $i: X^{*} \longrightarrow X^{*}$ such that $X$ becomes an imprimitivity bimodule between $\mathcal{L}$ and $\mathcal{R}$ under the pairings ${ }_{\mathcal{L}}\langle x, y\rangle=\left(x, i\left(y^{*}\right)\right)$ and $\langle x, y\rangle_{\mathcal{R}}=\left[i\left(x^{*}\right), y\right], x, y \in X$. It follows that, as a Banach space, $X$ is an imprimitivity bimodule in the sense of Rieffel. It only remains to be checked that the operator space structure of $X$ coincides with the canonical imprimitivity bimodule operator space structure $\left\{\|\cdot\|_{n}^{\prime}\right\}$ on $X$. By Lemma 5.6 of [2] we have that there are completely isometric representations $\sigma, \pi, \varphi, \psi$ of $\mathcal{L}, \mathcal{R}, X, X^{*}$ on appropriate Hilbert spaces such that if $\rho$ is the respective two by two matrix representation formed by $\sigma, \pi, \varphi$ and $\psi$, then $\rho$ is completely isometric and $\sigma\left(\left(x, y^{*}\right)\right)=$ $\varphi(x) \psi\left(y^{*}\right)$, for each $x, y \in X$. Let $x \in X$. Then 


$$
\begin{aligned}
\|x\|_{1}^{\prime 2} & =\left\|_{\mathcal{L}}\left\langle x, i\left(x^{*}\right)\right\rangle\right\|=\left\|\sigma\left(\left(x, i\left(x^{*}\right)\right)\right)\right\| \\
& =\left\|\varphi(x) \psi\left(i\left(x^{*}\right)\right)\right\|=\left\|\varphi(x) \varphi(x)^{*}\right\|=\|\varphi(x)\|^{2}=\|x\|^{2} .
\end{aligned}
$$

Thus, $\|\cdot\|^{\prime}=\|\cdot\|$. Note that for each $n \in \mathbb{N}$ the pair $\left(M_{n}(X), M_{n}\left(X^{*}\right)\right)$ is a Morita equivalence pair between the $\mathrm{C}^{*}$-algebras $M_{n}(\mathcal{L})$ and $M_{n}(\mathcal{R})$. Just as above we conclude that $\|\cdot\|_{n}^{\prime}=\|\cdot\|_{n}$.

The converse direction is obtained as the converse direction in Theorem 1.

Note that there was no need to impose a condition about existence of approximate units in Theorem 3: as we saw, the non-degeneracy condition (b) ensures that the algebras we construct are $\mathrm{C}^{*}$-algebras; a posteriori they are approximately unital or unital.

Theorem 3 leads us to the following definition.

Definition 4. A ternary operator system is an operator space $X$ equipped with a triple product $[\cdot, \cdot, \cdot]: X \times X \times X \longrightarrow X$, linear on the first and the third variable and conjugate linear on the second such that

(a) $\left[\left[x_{1}, x_{2}, x_{3}\right], x_{4}, x_{5}\right]=\left[x_{1},\left[x_{4}, x_{3}, x_{2}\right], x_{5}\right]=\left[x_{1}, x_{2},\left[x_{3}, x_{4}, x_{5}\right]\right]$ and

(b) $\left\|\left[\left(x_{i j}\right) \odot\left(x_{j i}\right) \odot\left(x_{i j}\right)\right]_{n}\right\|=\left\|\left(x_{i j}\right)\right\|_{n}^{3}$, for each $\left(x_{i j}\right) \in M_{n}(X), n \in \mathbb{N}$.

Recall that a ternary $\mathrm{C}^{*}$-ring is a Banach space endowed with a contractive triple product $[\cdot, \cdot, \cdot]: X \times X \times X \longrightarrow X$, linear on the first and the third variable and conjugate linear on the second, satisfying conditions (a) and (b) in Definition 4 for $n=1$. A consequence of Zettl's results is that the Banach spaces which are imprimitivity bimodules between certain $\mathrm{C}^{*}$-algebras are, up to isometry, precisely the ternary $\mathrm{C}^{*}$-rings. But, as we pointed out, an imprimitivity bimodule has a canonical structure of an operator space. Theorem 3 gives an operator space version of the above result: it states that the operator spaces which are imprimitivity bimodules bewteen certain $\mathrm{C}^{*}$-algebras are, up to a complete isometry, precisely the ternary operator systems. The conditions (a) and (b) for $n=1$ are not sufficient to ensure that the operator space is completely isometric to an imprimitivity bimodule - we must require moreover that condition (b) is fulfilled for every $n \in \mathbb{N}$. An example of an operator space $X$ which is a ternary $\mathrm{C}^{*}$-ring but not a ternary operator system is a $C^{*}$-algebra equipped with some non-canonical operator space structure.

Ternary $\mathrm{C}^{*}$-rings and ternary operator systems are the linear space analogue of the $\mathrm{C}^{*}$-algebras. A concrete version of these objects are the so called ternary rings of operators (TRO). Recall [6] that a TRO is a subspace $V \subseteq \mathcal{B}(H, K)$ of operators between two Hilbert spaces $H$ and $K$, closed under the (canonical) triple product $(a, b, c) \longrightarrow a b^{*} c$. Note that, as a concrete operator space, a TRO has a natural operator space structure. It is clear that a TRO is a ternary $\mathrm{C}^{*}$-ring and a ternary operator system with respect to the canonical triple product. A result analogous to the Gelfand-Naimark Theorem is proved in [6]: each ternary $\mathrm{C}^{*}$-ring $X$ has a decomposition $X=X_{+} \oplus X_{-}$such that $X_{+}$is (isometrically) isomorphic to a TRO while $X_{-}$is (isometrically) anti-isomorphic to a TRO. Of course, a morphism between ternary $C^{*}$-rings is defined as a bounded linear map that preserves the triple product. Similarly, a morphism between ternary operator systems is a completely bounded linear map that preserves the triple product. Theorem 3 has the following consequences. 
COROLlary 5. Let $X$ be a ternary operator system. Then there exist ternary operator sub-systems $X_{+}$and $X_{-}$such that $X=X_{+} \oplus X_{-}$and $X_{+}$is completely isometrically isomorphic to a TRO while $X_{-}$is completely isometrically anti-isomorphic to a TRO.

Thus, one is able to decompose a ternary operator system into parts on which the triple product can be given a concrete expression through Hilbert space operations. The triple product on the space $X_{+}$above is given, up to complete isometry, by $(a, b, c) \longrightarrow a b^{*} c$ while the triple product on $X_{-}$is given, again up to complete isometry, by $(a, b, c) \longrightarrow-a b^{*} c$.

If we are not interested in preserving the triple products, we can achieve the following representation.

COROllary 6. Let $X$ be a ternary operator system. Then $X$ is completely isometric to a TRO.

Acknowledgements. The author wishes to thank Aristides Katavolos for the useful discussions on the topic of this paper.

\section{REFERENCES}

1. D. P. Blecher, Z-j Ruan and A. M. Sinclair, A characterization of operator algebras, J. Functional Analysis, 89 (1990), 188-201.

2. D. P. Blecher, P. S. Muhly and V. I. Paulsen, Categories of operator modules (Morita equivalence and projective modules), Mem. Amer. Math. Soc., 143 (2000), no. 681.

3. E. Christensen and A. M. Sinclair, Representations of completely bounded multilinear operators, J. Functional Analysis 72 (1987), 151-181.

4. E. Effros and Z-j. Ruan, Operator spaces (Clarendon Press, Oxford, 2000).

5. M. Rieffel, Morita equivalence for $\mathrm{C}^{*}$-algebras and $\mathrm{W}^{*}$-algebras, J. Pure Appl. Algebra 5 (1974), 51-96. 143.

6. H. Zettl, A characterization of ternary rings of operators, Adv. Math, 48 (1983), 117 